\title{
Herschel-PACS observation of the 10 Myr old T Tauri disk TW Hya ${ }^{\star}$ Constraining the disk gas mass ${ }^{\star \star}$
}

W.-F. Thi ${ }^{1,2}$, G. Mathews ${ }^{3}$, F. Ménard ${ }^{2}$, P. Woitke ${ }^{4,5,1}$, G. Meeus ${ }^{6}$, P. Riviere-Marichalar ${ }^{7}$, C. Pinte ${ }^{2,8}$, C. D. Howard ${ }^{9}$, A. Roberge ${ }^{10}$, G. Sandell ${ }^{9}$, I. Pascucci ${ }^{11}$, B. Riaz ${ }^{11}$, C. A. Grady ${ }^{12}$, W. R. F. Dent ${ }^{13}$, I. Kamp ${ }^{14}$, G. Duchêne ${ }^{2,15}$, J.-C. Augereau ${ }^{2}$, E. Pantin ${ }^{16}$, B. Vandenbussche ${ }^{17}$, I. Tilling ${ }^{1}$, J. P. Williams ${ }^{3}$, C. Eiroa ${ }^{6}$, D. Barrado ${ }^{18,7}$, J. M. Alacid ${ }^{19,20}$, S. Andrews ${ }^{21}$, D. R. Ardila ${ }^{22}$, G. Aresu ${ }^{14}$, S. Brittain ${ }^{23}$, D. R. Ciardi ${ }^{24}$, W. Danchi25, D. Fedele ${ }^{26,27,28}$, I. de Gregorio-Monsalvo ${ }^{13}$, A. Heras ${ }^{29}$, N. Huelamo ${ }^{4}$, A. Krivov ${ }^{30}$, J. Lebreton ${ }^{2}$, R. Liseau ${ }^{31}$, C. Martin-Zaidi ${ }^{2}$, I. Mendigutía ${ }^{4}$, B. Montesinos ${ }^{4}$, A. Mora ${ }^{31}$, M. Morales-Calderon ${ }^{32}$, H. Nomura ${ }^{33}$, N. Phillips ${ }^{1}$, L. Podio ${ }^{14}$, D. R. Poelman ${ }^{5}$, S. Ramsay ${ }^{34}$, K. Rice ${ }^{1}$, E. Solano ${ }^{19,20}$, H. Walker ${ }^{35}$, G. J. White ${ }^{36,35}$, and G. Wright ${ }^{4}$

(Affiliations are available in the online edition)

Received 30 March 2010 / Accepted 28 April 2010

\section{ABSTRACT}

Planets are formed in disks around young stars. With an age of $\sim 10 \mathrm{Myr}$, TW Hya is one of the nearest T Tauri stars that is still surrounded by a relatively massive disk. In addition a large number of molecules has been found in the TW Hya disk, making TW Hya the perfect test case in a large survey of disks with Herschel-PACS to directly study their gaseous component. We aim to constrain the gas and dust mass of the circumstellar disk around TW Hya. We observed the fine-structure lines of [O I] and [C II] as part of the open-time large program GASPS. We complement this with continuum data and ground-based ${ }^{12} \mathrm{CO} 3-2$ and ${ }^{13} \mathrm{CO} 3-2$ observations. We simultaneously model the continuum and the line fluxes with the 3D Monte-Carlo code MCFOST and the thermo-chemical code ProDiMo to derive the gas and dust masses. We detect the [O I] line at $63 \mu \mathrm{m}$. The other lines that were observed, [OI] at $145 \mu \mathrm{m}$ and [C II] at $157 \mu \mathrm{m}$, are not detected. No extended emission has been found. Preliminary modeling of the photometric and line data assuming $\left[{ }^{12} \mathrm{CO}\right] /\left[{ }^{13} \mathrm{CO}\right]=69$ suggests a dust mass for grains with radius $<1 \mathrm{~mm}$ of $\sim 1.9 \times 10^{-4} M_{\odot}$ (total solid mass of $3 \times 10^{-3} M_{\odot}$ ) and a gas mass of $(0.5-5) \times 10^{-3} M_{\odot}$. The gas-to-dust mass may be lower than the standard interstellar value of 100 .

Key words. circumstellar disks

\section{Introduction}

Planets are formed in the disks that surround a large fraction of $\mathrm{T}$ Tauri stars. Knowledge of the gas mass available at different disk ages is essential to constrain giant planet formation models. Most studies estimate the dust mass from millimeter continuum emission and assume the gas mass is a factor of 100 times larger. This conversion factor has been calibrated for the interstellar medium but is likely not valid for disks, especially those that are evolving toward debris disks or where most of the gas has accreted onto the planetary atmosphere. Disk gas mass estimates derived from observations of ${ }^{12} \mathrm{CO}$ and optically thinner ${ }^{13} \mathrm{CO}$ emission are at least a factor of 10 lower than the mass derived from dust observations assuming the interstellar medium conversion factor. The discrepancy has been ascribed to $\mathrm{CO}$ photodissociation at disk atmosphere and freeze-out onto cold dust grains in the disk midplane (e.g., Qi et al. 2004; Thi et al. 2001). An alternative explanation is that the $\mathrm{CO}$ abundance is not different and the gas in disks has been depleted.

\footnotetext{
* Herschel is an ESA space observatory with science instruments provided by Principal Investigator consortia. It is open for proposals for observing time from the worldwide astronomical community.

$\star \star$ Appendix is only available in electronic form at http://www . aanda. org
}

The PACS instrument (Poglitsch et al. 2010) on-board the Herschel Space Observatory (Pilbratt et al. 2010) makes it possible to observe lines from species that result from the photodissociation of $\mathrm{CO}$ (atomic oxygen and singly ionized carbon). With observations of all the major gas-phase carbon and oxygenbearing species, we can more precisely constrain the disk gas mass.

At a distance of $~ 56$ pc (Wichmann et al. 1998), TW Hya is one of the nearest classical T Tauri stars with an estimated age of 10 Myr (Barrado Y Navascués 2006). Its proximity allows us to attain an order of magnitude higher mass sensitivity than objects in the Taurus molecular cloud. Fits to the spectral energy distribution (SED) provide an estimate of the gas disk mass of $6 \times 10^{-2} M_{\odot}$ after applying a conversion factor of $\sim 75$ (Calvet et al. 2002). This large disk mass at this advanced age is surprising as the median disk lifetime is only 2-3 Myr (Haisch et al. 2001). TW Hya is considered a transition object with an optically thin inner cavity and an optically thick outer disk (Calvet et al. 2002; Ratzka et al. 2007). The fit to the SED also suggests that grains have grown to at least $\sim 1 \mathrm{~cm}$.

The star TW Hya was observed as a science demonstration program object and is part of the Herschel-GASPS program (Dent \& GASPS team 2010). Herschel observations of the disk around the Herbig Ae star HD169142 are presented by Meeus et al. (2010). In this letter we use fine-structure lines in addition 
Table 1. Lines observed by Herschel-PACS.

\begin{tabular}{|c|c|c|c|c|}
\hline Line & $\begin{array}{c}\text { Cont. flux } \\
\text { (Jy) }\end{array}$ & $\begin{array}{l}\text { Obs. } \\
\qquad\left(10^{-1}\right.\end{array}$ & $\begin{array}{r}\mathrm{GH} 08 \\
\left.\mathrm{~W} \mathrm{~m}^{-2}\right) \\
\end{array}$ & M08 \\
\hline OI ${ }^{3} \mathrm{P}_{1} \rightarrow{ }^{3} \mathrm{P}_{2}$ & $2.99 \pm 0.14$ & $36.5 \pm 12.1$ & $124-161$ & 412 \\
\hline OI ${ }^{3} \mathrm{P}_{0} \rightarrow{ }^{3} \mathrm{P}_{1}$ & $7.00 \pm 0.05$ & $<5.5$ & $25-41$ & 11 \\
\hline $\mathrm{CII}{ }^{2} \mathrm{P}_{3 / 2} \rightarrow{ }^{2} \mathrm{P}_{1 / 2}$ & $8.79 \pm 0.08$ & $<6.6$ & $0.8-12$ & 0.06 \\
\hline $\mathrm{CO} 3-2$ & n.a. & 0.43 & $0.3-0.6$ & n.a. \\
\hline${ }^{13} \mathrm{CO} 3-2$ & n.a. & $4.4 \times 10^{-2}$ & n.a. & n.a. \\
\hline
\end{tabular}

Notes. The errors and upper limits are $3 \sigma$. The calibration error adds an extra $\sim 40 \%$ uncertainty. The CO data also have uncertainties of $30 \%$.

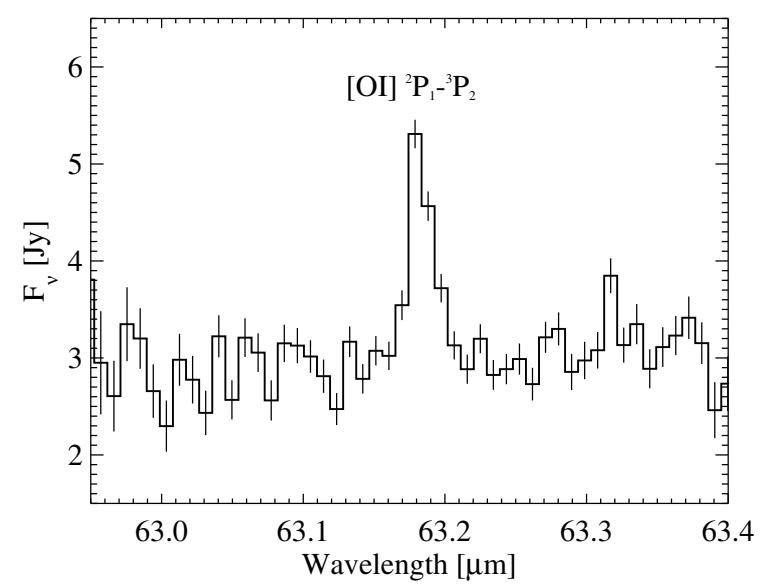

Fig. 1. Herschel-PACS spectrum centred around the OI $63 \mu \mathrm{m}$ line on the upper-left panel.

to continuum data and $\mathrm{CO}$ (sub)millimeter lines to directly constrain the gas mass and compare it to the dust mass derived from fits to the SED.

\section{Observations and results}

We obtained photometry in the "blue" $(70 \mu \mathrm{m})$ and "red" $(160 \mu \mathrm{m})$ band of the PACS camera by doing mini scan maps with a scan speed of $20^{\prime \prime}$ and a scan length of $2^{\prime}$ (obsid 1342187342). The total duration of this map was $731 \mathrm{~s}$, with an on-source time of $146 \mathrm{~s}$. The results are $3.90 \pm 0.02 \mathrm{Jy}$ and $7.38 \pm 0.04$ in the blue and red band respectively and have an absolute accuracy estimated to be $5 \%$ for the blue channel and $10 \%$ for the the red channel. These values agree very well with the observed IRAS flux densities and also with the continuum flux densities measured with the PACS spectrometer (Table 1). We also used the PACS spectrometer to target the [OI] line at $63 \mu \mathrm{m}$ in line scan mode, and the [OI] and [CII] lines at 145 and $158 \mu \mathrm{m}$, respectively in range scan mode (obsid 1342187127 PacsLineSpec and obsid 1342187238 PacsRangeSpec). Only the [OI] line at $63 \mu \mathrm{m}$ was detected and we report upper limits for the other two lines; see Table 1 . The absolute accuracy of PACS spectroscopy is currently estimated to be about $40 \%$, but is expected to improve in the future. Figure 1 shows the spectrum centered at the position of the OI line at $63 \mu \mathrm{m}$ of the central pixel.

\section{Modeling and discussion}

As there is no evidence for an outflow from TW Hya, we assume that all the fluxes arise from the circumstellar disk (see also the discussion in Mathews et al. 2010). The interpretation
Table 2. Disk parameters for the modeling.

\begin{tabular}{|c|c|c|c|}
\hline \multicolumn{4}{|c|}{ Fixed parameters } \\
\hline & & \multicolumn{2}{|c|}{ Inner cavity Outer ring } \\
\hline Stellar mass & $M_{*}\left(M_{\odot}\right)$ & \multicolumn{2}{|c|}{0.6} \\
\hline Stellar luminosity & $L_{*}\left(L_{\odot}\right)$ & \multicolumn{2}{|c|}{0.23} \\
\hline Effective temperature & $T_{\text {eff }}(\mathrm{K})$ & \multicolumn{2}{|c|}{4000} \\
\hline Solid material mass density & $\rho_{\text {dust }}\left(\mathrm{g} \mathrm{cm}^{-3}\right)$ & \multicolumn{2}{|c|}{3.5} \\
\hline Inner radius & $R_{\text {in }}(\mathrm{AU})$ & 0.25 & 4 \\
\hline Outer radius & $R_{\text {out }}(\mathrm{AU})$ & 4 & 196 \\
\hline ISM UV field & $\chi$ & \multicolumn{2}{|c|}{1.0} \\
\hline$\alpha$ viscosity parameter & $\alpha$ & \multicolumn{2}{|c|}{0.0} \\
\hline Turbulent velocity & $v_{\text {turb }}\left(\mathrm{km} \mathrm{s}^{-1}\right)$ & \multicolumn{2}{|c|}{0.05} \\
\hline Disk inclination & $i$ & \multicolumn{2}{|c|}{7} \\
\hline $\mathrm{CO}$ isotopologue ratio & {$\left[{ }^{12} \mathrm{CO}\right] /\left[{ }^{13} \mathrm{CO}\right]$} & \multicolumn{2}{|c|}{69} \\
\hline \multicolumn{4}{|c|}{$M C F O S T$ best fit parameters } \\
\hline Column density index & $\epsilon$ & \multicolumn{2}{|c|}{1} \\
\hline Reference scale height & $H_{0}(\mathrm{AU})$ & 2.0 & 10.0 \\
\hline Reference radius & & 100 & 100 \\
\hline Flaring index & $\gamma$ & 0.6 & 1.12 \\
\hline Minimum grain size & $a_{\min }(\mu \mathrm{m})$ & \multicolumn{2}{|c|}{$3 \times 10^{-2}$} \\
\hline Maximum grain size & $a_{\max }(\mathrm{cm})$ & \multicolumn{2}{|c|}{10} \\
\hline Dust size distribution index & $p$ & \multicolumn{2}{|c|}{3.4} \\
\hline Dust mass $(a<1 \mathrm{~mm})$ & $M_{\text {dust }}\left(M_{\odot}\right)$ & $1.2 \times 10^{-9}$ & $1.9 \times 10^{-4}$ \\
\hline Solid mass & $M_{\text {solid }}\left(M_{\odot}\right)$ & $2.0 \times 10^{-8}$ & $3.0 \times 10^{-3}$ \\
\hline \multicolumn{4}{|c|}{ ProDiMo parameter range } \\
\hline Disk gas mass & $M_{\text {gas }}\left(M_{\odot}\right)$ & \multicolumn{2}{|c|}{$3 \times 10^{-4}-0.3$} \\
\hline UV excess & $F_{\mathrm{UV}}$ & \multicolumn{2}{|c|}{0.018} \\
\hline Fraction of PAHs w.r.t. ISM & $f_{\mathrm{PAH}}$ & \multirow{2}{*}{\multicolumn{2}{|c|}{$\begin{array}{c}0.01,0.1 \\
(1.7-17) \times 10^{-17}\end{array}$}} \\
\hline Cosmic ray flux & $\zeta\left(\mathrm{s}^{-1}\right)$ & & \\
\hline
\end{tabular}

of the observations with the DENT grid of models is detailed in Pinte et al. (2010). We performed a more detailed analysis here.

We first augmented the Herschel photometric data with continuum measurements from the literature. We also retrieved and reduced archival SCUBA data for TW Hya obtained during two nights with very good sub-millimeter transmission $\left(F_{\nu}(450 \mu \mathrm{m})=4.25 \pm 0.85 \mathrm{Jy}\right.$ and $\left.F_{v}(850 \mu \mathrm{m})=1.38 \pm 0.14 \mathrm{Jy}\right)$. The disk around TW Hya has an internal cavity from up to 4 AU where the gas and dust density are very low. Most of the mass is located in the external ring. The inner $\left(R_{\mathrm{in}}\right)$ and outer radius $\left(R_{\text {out }}\right)$ of the external ring are well constrained by imaging studies and are fixed at $4 \mathrm{AU}$ and $200 \mathrm{AU}$ respectively (Roberge et al. 2005; Qi et al. 2004; Hughes et al. 2007). We fitted the SED with the 3D Monte-Carlo radiative transfer code MCFOST (Pinte et al. 2006). We chose to restrict to a parametric disk model for this letter. The disk has a radial density profile with index $\epsilon$. The flaring is characterized by an opening angle $H_{0}$ at a given radius $R_{\text {ref }}$ and a flaring index $\gamma$ so that the gas scale-height is given by $H=H_{0}\left(R / R_{\text {ref }}\right)^{\gamma}$. The low continuum flux in the $30-100 \mu \mathrm{m}$ region suggests that the outer disk flaring is weak. Amorphous olivine grains were used (Dorschner et al. 1995) with a power-law size-distribution defined by a minimum radius $a_{\text {min }}$, maximum radius $a_{\max }$, and power-law index $p$. The dust size-distribution and mass are well constrained by the continuum emission at long wavelengths. The fit to the long-wavelength photometric points including the new Herschel-PACS data is shown in Fig. 2 and the disk parameters constrained by the fit are listed in Table 2. The inferred dust mass in grains with radius $<1 \mathrm{~mm}$ is $M_{\text {dust }}=1.9 \times 10^{-4} M_{\odot}$ and the total mass in solids (pebbles) up to $a_{\max }=10 \mathrm{~cm}$ is $M_{\text {solid }}=3 \times 10^{-3} M_{\odot}$. However, the fit fails to account for the flux at $\sim 25 \mu \mathrm{m}$, which may 


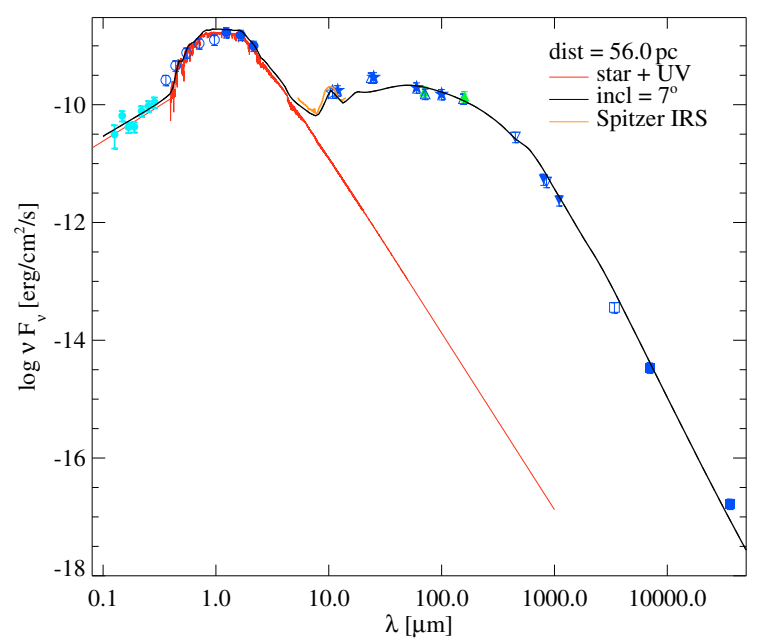

Fig. 2. Fit to the SED generated by ProDiMo using the parameters from MCFOST. The input Phoenix stellar spectrum plotted in red is from Brott \& Hauschildt (2005). IUE (UV) data are from Valenti et al. (2003). The $2 M A S S J, H, K, I R A S$, and Spitzer-MIPS photometry are archival data. The Spitzer-IRS spectrum is published by Ratzka et al. (2007). The Herschel-PACS data are plotted in filled green triangles. The average $U B V R I$ photometric points are published by Rucinski \& Krautter (1983). The $800 \mu \mathrm{m}$ and $1.1 \mathrm{~mm}$ data points (inverted filled blue triangles) are taken from Weintraub et al. (1989). The $3.4 \mathrm{~mm}$ point (open blue square) is from Wilner et al. (2003) while the $7 \mathrm{~mm}$ and $3.6 \mathrm{~cm}$ points (filled blue square) are from Wilner et al. (2000).

stem from our assumption of a unique temperature for grains of all sizes. The flux around $20-30 \mu \mathrm{m}$ is strongly inclinationdependent because we adopted a sharp density change between the inner cavity and the outer ring at 4 AU. Solids as large as $10 \mathrm{~cm}$ in radius are needed to account for the observed $7 \mathrm{~mm}$ and $3.6 \mathrm{~cm}$ flux (Wilner et al. 2000). The small grains in the TW Hya disk account for $6 \%$ of the total solid mass. We also estimated a mass in small grains of $3 \times 10^{-4} M_{\odot}$ assuming that the emission in the millimeter is optically thin, an average dust temperature of $20 \mathrm{~K}$, and grain opacity $\kappa_{v}=2.0\left(v / v_{0}\right)^{-\beta} \mathrm{g} \mathrm{cm}^{-2}$ where $v_{0}=230.769 \mathrm{GHz}$ and $\beta=0.6$ (Beckwith \& Sargent 1991). The two estimates of dust mass (with radius $a<1 \mathrm{~mm}$ ) are consistent within a factor 2 with each other. The visibility amplitudes generated by the models are consistent with the observed amplitudes at $345 \mathrm{GHz}$ by Qi et al. (2004).

For the line observations we augmented the Herschel data with SMA CO 3-2 (Qi et al. 2004) and JCMT ${ }^{13} \mathrm{CO} 3-2 \mathrm{ob}-$ servations (Thi et al. 2004). Following the characterization of the disk structure from the SED, we ran three series of models with the thermo-chemical code ProDiMo (a detailed description is given in Woitke et al. 2009). In ProDiMo species abundances are computed at steady-state from the gas, and dust temperature as well as the local UV field for the photodissociation reactions. A constant isotopologue ratio $\left[{ }^{13} \mathrm{CO}\right] /\left[{ }^{12} \mathrm{CO}\right]$ of 69 is assumed. The gas kinetic temperature is computed by balancing heating and cooling processes. Line profiles are computed by non-LTE radiative transfer within ProDiMo. The disk is assumed to be passively heated. The disk turbulent velocity and inclination are well constrained by millimeter interferometric data (Qi et al. 2004). The outer disk is irradiated by direct and scattered stellar photons as well as by interstellar UV photons. The free parameters of the gas simulations are the disk gas mass $M_{\text {gas }}$ (between $3 \times 10^{-4}$ and $0.3 M_{\odot}$ ), the fraction of polycyclic aromatic hydrocarbons (PAHs) in the disk with respect to the interstellar abundance $f_{\mathrm{PAH}}$, and the cosmic ray flux $\zeta$ $\left(=1.7 \times 10^{-17} \mathrm{~s}^{-1}\right.$ in the ISM). Observations show that PAHs are depleted by at least a factor of $10\left(f_{\mathrm{PAH}}=0.1\right)$ in disks with respect to the interstellar abundance (Geers et al. 2006). Because the gas is mostly heated by photoelectrons ejected from $\mathrm{PAH}$, the $\mathrm{PAH}$ abundance is the main free parameter that controls the gas temperature. The three series of models correspond to three possible states: disks with a very low PAH abundance $\left(f_{\mathrm{PAH}}=0.01\right)$, disks with a typical PAH abundance $\left(f_{\mathrm{PAH}}=0.1\right)$, and X-ray irradiated disks with a low PAH abundance $\left(f_{\mathrm{PAH}}=0.01\right)$ but ten times the standard cosmic ray flux $\left(\zeta=1.7 \times 10^{-17} \mathrm{~s}^{-1}\right)$ to mimic the influence of strong X-ray emission (Bruderer et al. 2009). The model results are plotted in Fig. 3. The density, dust and gas temperature structure are shown for a typical disk in the appendix. The results from series 3 are within $10 \%$ of the values of series 2, suggesting that X-ray does not influence the line fluxes that are emitted at radii beyond a few AU. In panels a and b we can see that the OI $63 \mu \mathrm{m}$ and $145 \mu \mathrm{m}$ flux increases with the disk gas mass. The OI $63 \mu \mathrm{m}$ line is optically thick while the OI $145 \mu \mathrm{m}$ line is optically thin for all models. Both lines arise mostly in a ring between 4 and $10 \mathrm{AU}$ and thus probe the gas mass up to 10-20 AU with 10-20\% contribution from the inner cavity (panel f). In panel c the CII flux first starts to increase with higher disk gas mass but then plummets for disk gas masses greater than $10^{-2} M_{\odot}$. The CII line is optically thin and the flux increases with radius. As the disk becomes more massive, more carbon is converted into $\mathrm{CO}$ and the disk becomes cooler. Ionized carbon is excited in gas at $\sim 100 \mathrm{~K}$. The CO 3-2 flux increases with increasing disk gas mass although the emission line is highly optically thick with $\tau>100$ (panel d). CO 3-2 emission comes from the outer disk $(R>50 \mathrm{AU})$. Finally, panel e illustrates the use of the line emission ratio between two isotopologues $\left({ }^{12} \mathrm{CO}\right.$ and $\left.{ }^{13} \mathrm{CO}\right)$ to constrain column densities or masses. The flux difference between the two isotopologues shrinks with increasing disk gas mass. The observed ${ }^{12} \mathrm{CO} /{ }^{13} \mathrm{CO}$ 3-2 ratio is consistent with a very low-mass disk. The CII and CO lines probe the outer disk mass (panel f). All together, the observations constrain the disk gas mass between $5 \times 10^{-4}$ and $5 \times 10^{-3} M_{\odot}$.

The modeling suggests that the disk of TW Hya has a gasto-dust mass ratio of 2.6-26, around a factor of 10 lower than the interstellar value. If we compare the gas mass to the total mass in solids (ie including solids with radii up to $10 \mathrm{~cm}$ ), the gas-to-solid ratio is 0.17-1.7. Gorti \& Hollenbach (2008, GH08) included X-ray and UV heating in modeling the disk of TW Hya with a gas mass of $0.03 M_{\odot}$ but noticed that X-ray weakly influences the fluxes. Their model overestimates the two OI line fluxes (Table 1 and Fig. 3). Disk models with X-ray heating only also predict too strong OI fluxes (Meijerink et al. 2008, M08) for their model with $L_{X}=2 \times 10^{30} \mathrm{erg} \mathrm{s}^{-1}$ scaled to $d=56 \mathrm{pc}$. At $\sim 10 \mathrm{Myr}$, TW Hya is one of the oldest classical T Tauri stars. The outer dust disk is very long-lived, while the inner disk contains little amount of material. The gas may have a shorter lifetime than the dust due to photoevaporation or the small grains result from collisions between the large grains. TW Hya is one of the strongest X-ray active T Tauri stars (Raassen 2009), which may result in a high gas photoevaporation rate (Owen et al. 2010) as evidenced in the blueshifted [Ne II] emission observed by Pascucci \& Sterzik (2009).

\section{Conclusion}

The Herschel-PACS spectral observations were used to constrain the gas disk mass surrounding the $10 \mathrm{Myr} \mathrm{T}$ Tauri star TW Hya. We estimate the gas mass to be $(0.5-5) \times 10^{-3} M_{\odot}$ compared to the dust mass $\left(a_{\max }<1 \mathrm{~mm}\right)$ of $1.9 \times 10^{-4} M_{\odot}$. The 
A\&A 518, L125 (2010)
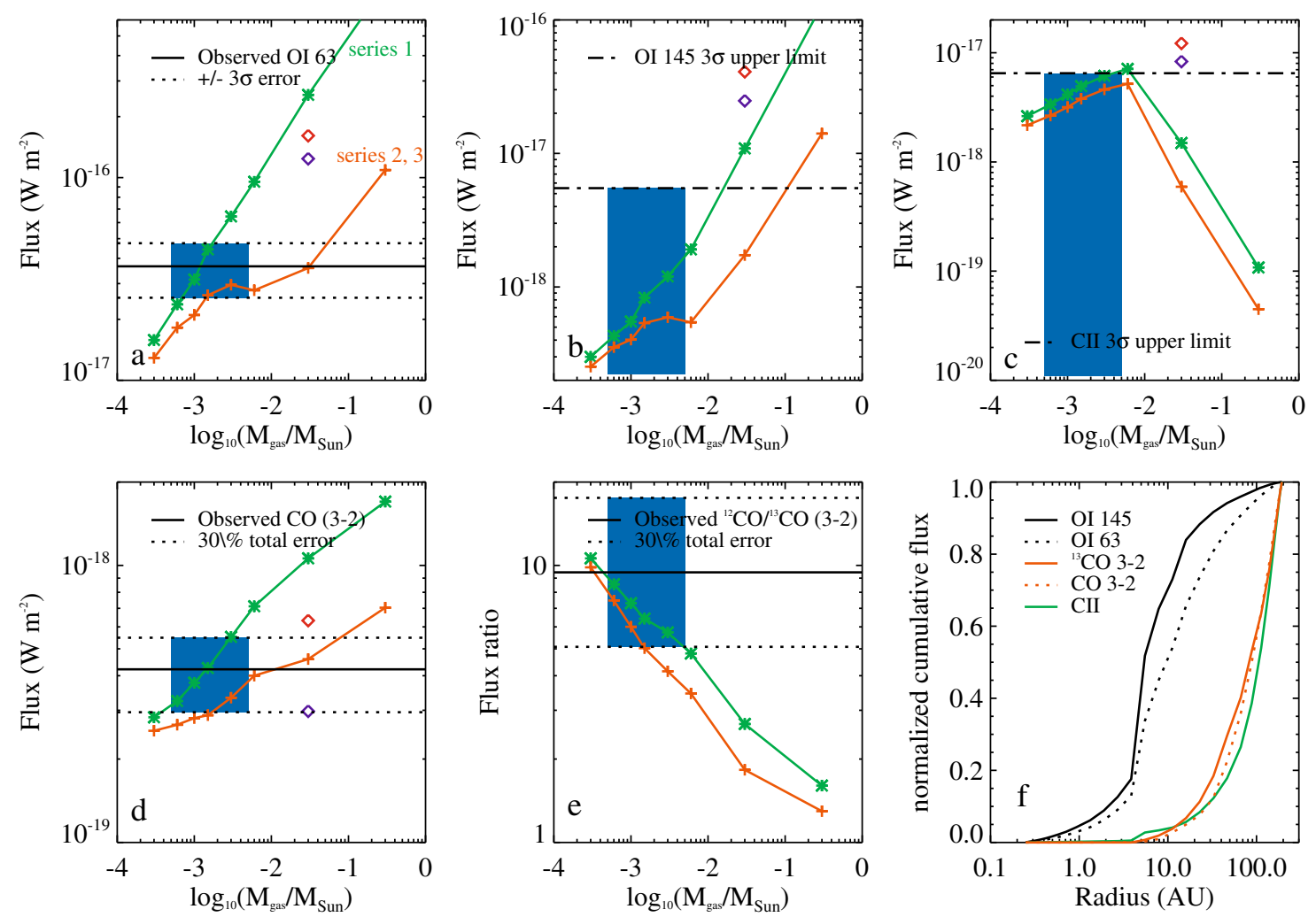

Fig. 3. Three series of model results compared to observations. The blue boxes enclose the model outputs for disk gas mass between $5 \times 10^{-4} M_{\odot}$ and $5 \times 10^{-3} M_{\odot}$. Panel a) shows the predictions and observation of the OI $63 \mu \mathrm{m}$ line. The $3 \sigma$ uncertainty range is plotted as dashed lines. Panels b) and c) show the predicted fluxes and the $3 \sigma$ upper limits for the OI $145 \mu \mathrm{m}$ and CII lines. The two lower panels (d) and e)) are the comparison between observations and model outputs for ${ }^{12} \mathrm{CO} 3-2$ emission and the ${ }^{12} \mathrm{CO} /{ }^{13} \mathrm{CO} 3-2$ ratio. Panel f) shows the normalized cumulative fluxes for a $10^{-3} M_{\odot}$ model (series 1 ). The diamonds ( $\diamond R_{\text {out }}=174$ AU model, $\diamond R_{\text {out }}=120$ AU model) show the predictions for TW Hya from GH08.

gas-to-dust mass ratio is $\sim 2.6-26$, lower than the standard interstellar value of 100 . The ratio gas-to-total-mass in solids is 0.17-1.7. Although the disk is still massive, a significant fraction of the primordial gas has already disappeared. A large fraction of the primordial gas may have been evaporated due to the strong X-ray flux from TW Hya. TW Hya is the first example where the disk gas mass around a transitional $\mathrm{T}$ Tauri star can be determined accurately and directly from gas phase lines. However, more detailed modeling that includes X-ray physics and ${ }^{13} \mathrm{CO}$ photochemistry is needed to confirm the low gas mass.

Acknowledgements. W.-F. Thi acknowledges a SUPA astrobiology fellowship. G. Meeus, C. Eiroa, J. Maldonado and B. Montesinos are partly supported by Spanish grant AYA 2008-01727. C. Pinte acknowledges the funding from the EC 7th Framework Program as a Marie Curie Intra-European Fellow (PIEF-GA2008-220891). D.R. Ardila, S.D. Brittain, C.A. Grady, I. Pascucci, B. Riaz, G. Sandell and C.D. Howards, J.-P. Williams, G. Matthews, A. Roberge, W. Danchi acknowledge NASA/JPL for funding support. E. Solano and J.M. Alacid acknowledge the funding from the Spanish MICINN through grant AYA200802156. The LAOG group acknowledges PNPS, CNES and ANR (contract ANR07-BLAN-0221) for financial support.

\section{References}

Barrado Y Navascués, D. 2006, A\&A, 459, 511

Beckwith, S. V. W., \& Sargent, A. I. 1991, ApJ, 381, 250

Brott, I., \& Hauschildt, P. H. 2005, in The Three-Dimensional Universe with Gaia, ed. C. Turon, K. S. O'Flaherty, \& M. A. C. Perryman, ESA Special Publication, 576, 565
Bruderer, S., Doty, S. D., \& Benz, A. O. 2009, ApJS, 183, 179

Calvet, N., D’Alessio, P., Hartmann, L., et al. 2002, ApJ, 568, 1008 Dent, W. R. F., \& GASPS team. 2010, PASP

Dorschner, J., Begemann, B., Henning, T., et al. 1995, A\&A, 300, 503 Geers, V. C., Augereau, J., Pontoppidan, K. M., et al. A\&A, 459, 545 Gorti, U., \& Hollenbach, D. 2008, ApJ, 683, 287

Haisch, Jr., K. E., Lada, E. A., \& Lada, C. J. 2001, ApJ, 553, L153

Hughes, A. M., Wilner, D. J., Calvet, N., et al. ApJ, 664, 536

Mathews, G. S., et al. 2010, A\&A, 518, L127

Meeus, G., et al. 2010, A\&A, 518, L124

Meijerink, R., Glassgold, A. E., \& Najita, J. R. 2008, ApJ, 676, 518 Owen, J. E., Ercolano, B., Clarke, C. J., et al. 2010, MNRAS, 401, 1415 Pascucci, I., \& Sterzik, M. 2009, ApJ, 702, 724

Pilbratt, G. L., et al. 2010, A\&A, 518, L1

Pinte, C., Ménard, F., Duchêne, G., \& Bastien, P. 2006, A\&A, 459, 797

Pinte, C., et al. 2010, A\&A, 518, L126

Poglitsch, A., et al. 2010, A\&A, 518, L2

Qi, C., Ho, P. T. P., Wilner, D. J., et al. 2004, ApJ, 616, L11

Raassen, A. J. J. 2009, A\&A, 505, 755

Ratzka, T., Leinert, C., Henning, T., et al. 2007, A\&A, 471, 173

Roberge, A., Weinberger, A. J., \& Malumuth, E. M. 2005, ApJ, 622, 1171

Rucinski, S. M., \& Krautter, J. 1983, A\&A, 121, 217

Thi, W. F., van Dishoeck, E. F., Blake, G. A., et al. 2001, ApJ, 561, 1074 Thi, W., van Zadelhoff, G., \& van Dishoeck, E. F. 2004, A\&A, 425, 955 Valenti, J. A., Fallon, A. A., \& Johns-Krull, C. M. 2003, ApJS, 147, 305 Weintraub, D. A., Sandell, G., \& Duncan, W. D. 1989, ApJ, 340, L69 Wichmann, R., Bastian, U., Krautter, J., et al. 1998, MNRAS, 301, L39 Wilner, D. J., Bourke, T. L., Wright, C. M., et al. 2003, ApJ, 596, 597 Wilner, D. J., Ho, P. T. P., Kastner, J. H., \& Rodríguez, L. F. 2000, ApJ, 534, L101

Woitke, P., Kamp, I., \& Thi, W. 2009, A\&A, 501, 383

Pages 5 to 6 are available in the electronic edition of the journal at htttp://www . aanda.org 
1 SUPA, Institute for Astronomy, University of Edinburgh, Royal Observatory Edinburgh, UK

e-mail: wfdt@roe.ac.uk

2 Université Joseph-Fourier Grenoble 1/CNRS, Laboratoire d'Astrophysique de Grenoble (LAOG) UMR 5571, BP 53, 38041 Grenoble Cedex 09, France

3 Institute for Astronomy, University of Hawaii at Manoa, Honolulu, HI 96822, USA

${ }^{4}$ UK Astronomy Technology Centre, Royal Observatory, Edinburgh, Blackford Hill, Edinburgh EH9 3HJ, UK

5 School of Physics \& Astronomy, University of St. Andrews, North Haugh, St. Andrews KY16 9SS, UK

${ }^{6}$ Dep. de Física Teórica, Fac. de Ciencias, UAM Campus Cantoblanco, 28049 Madrid, Spain

7 LAEX, Depto. Astrofísica, Centro de Astrobiología (INTA-CSIC), PO Box 78, 28691 Villanueva de la Cañada, Spain

8 School of Physics, University of Exeter, Stocker Road, Exeter EX4 4QL, UK

9 SOFIA-USRA, NASA Ames Research Center, Mailstop 211-3 Moffett Field CA 94035, USA

10 Exoplanets and Stellar Astrophysics Lab, NASA Goddard Space Flight Center, Code 667, Greenbelt, MD, 20771, USA

11 Space Telescope Science Institute, 3700 San Martin Drive, Baltimore, MD 21218, USA

12 Eureka Scientific and Exoplanets and Stellar Astrophysics Lab, NASA Goddard Space Flight Center, Code 667, Greenbelt, MD, 20771, USA

13 ESO-ALMA, Avda Apoquindo 3846, Piso 19, Edificio Alsacia, Las Condes, Santiago, Chile

14 Kapteyn Astronomical Institute, PO Box 800, 9700 AV Groningen, The Netherlands

15 Astronomy Department, University of California, Berkeley CA 94720-3411, USA

16 CEA/IRFU/SAp, AIM UMR 7158, 91191 Gif-sur-Yvette, France

17 Instituut voor Sterrenkunde, KU Leuven, Celestijnenlaan 200D, 3001 Leuven, Belgium

18 Calar Alto Observatory, Centro Astronómico Hispano-Alemán C/Jesús Durbán Remón, 2-2, 04004 Almería, Spain
19 Unidad de Archivo de Datos, Depto. Astrofísica, Centro de Astrobiología (INTA-CSIC), PO Box 78, 28691 Villanueva de la Cañada, Spain

20 Spanish Virtual Observatory

21 Harvard-Smithsonian Center for Astrophysics, 60 Garden St., Cambridge, MA, USA

22 NASA Herschel Science Center, California Institute of Technology, Pasadena, CA, USA

${ }^{23}$ Clemson University, Clemson, SC, USA

24 NASA Exoplanet Science Institute/Caltech 770 South Wilson Avenue, Mail Code: 100-22, Pasadena, CA USA 91125, USA

25 NASA Goddard Space Flight Center, Exoplanets \& Stellar Astrophysics, Code 667, Greenbelt, MD 20771, USA

26 Departamento de Fisica Teórica, Facultad de Ciencias, Universidad Autónomade Madrid, Cantoblanco, 28049 Madrid, Spain

27 Max Planck Institut für Astronomie, Königstuhl 17, 69117 Heidelberg, Germany

28 Johns Hopkins University Dept. of Physics and Astronomy, 3701 San Martin drive Baltimore, MD 21210, USA

29 Research and Scientific Support Department-ESA/ESTEC, PO Box 299, 2200 AG Noordwijk, The Netherlands

30 Astrophysikalisches Institut und Universitätssternwarte, FriedrichSchiller-Universität, Schillergäßchen 2-3, 07745 Jena, Germany

31 Department of Radio and Space Science, Chalmers University of Technology, Onsala Space Observatory, 43992 Onsala, Sweden

32 ESA-ESAC Gaia SOC, PO Box 78. 28691 Villanueva de la Cañada, Madrid, Spain

33 Department of Astronomy, Graduate School of Science, Kyoto University, Kyoto 606-8502, Japan

34 European Southern Observatory, Karl-Schwarzschild-Strasse, 2, 85748 Garching bei München, Germany

35 The Rutherford Appleton Laboratory, Chilton, Didcot, OX11 OQL, UK

36 Department of Physics \& Astronomy, The Open University, Milton Keynes MK7 6AA, UK and The Rutherford Appleton Laboratory, Chilton, Didcot, OX11 OQL, UK 


\section{Appendix A: Density and temperature structure}

We show in Figs. A.1, A.2, and A.3 the density, dust temeprature, and gas temperature profile respectively for a disk model with $M_{\text {gas }}=2.9 \times 10^{-3} M_{\odot}, f_{\mathrm{PAH}}=0.1$, and $\zeta=1.7 \times 10^{-17} \mathrm{~s}^{-1}$. All other parameters are given in Table 2.

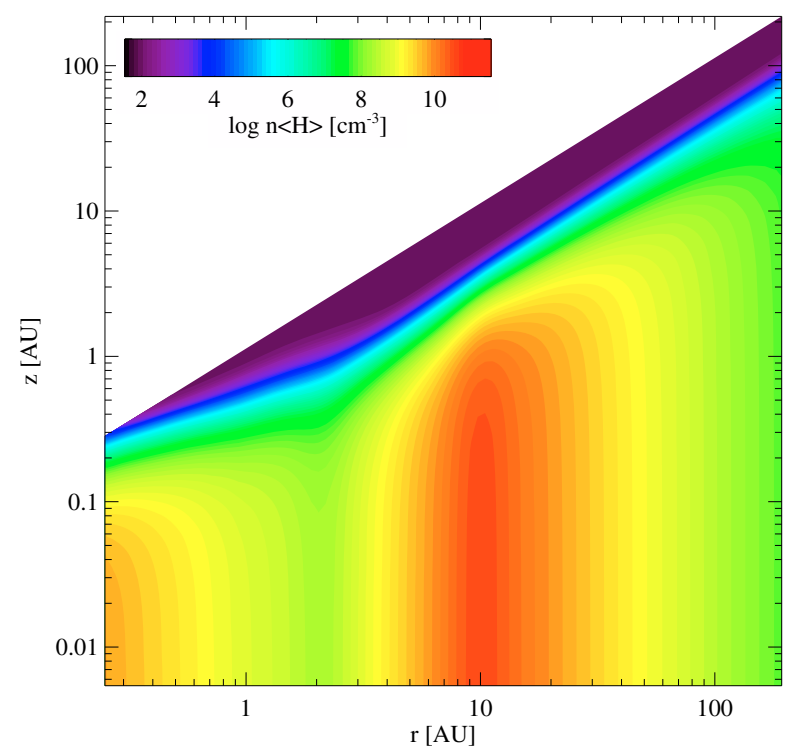

Fig. A.1. Density profile.

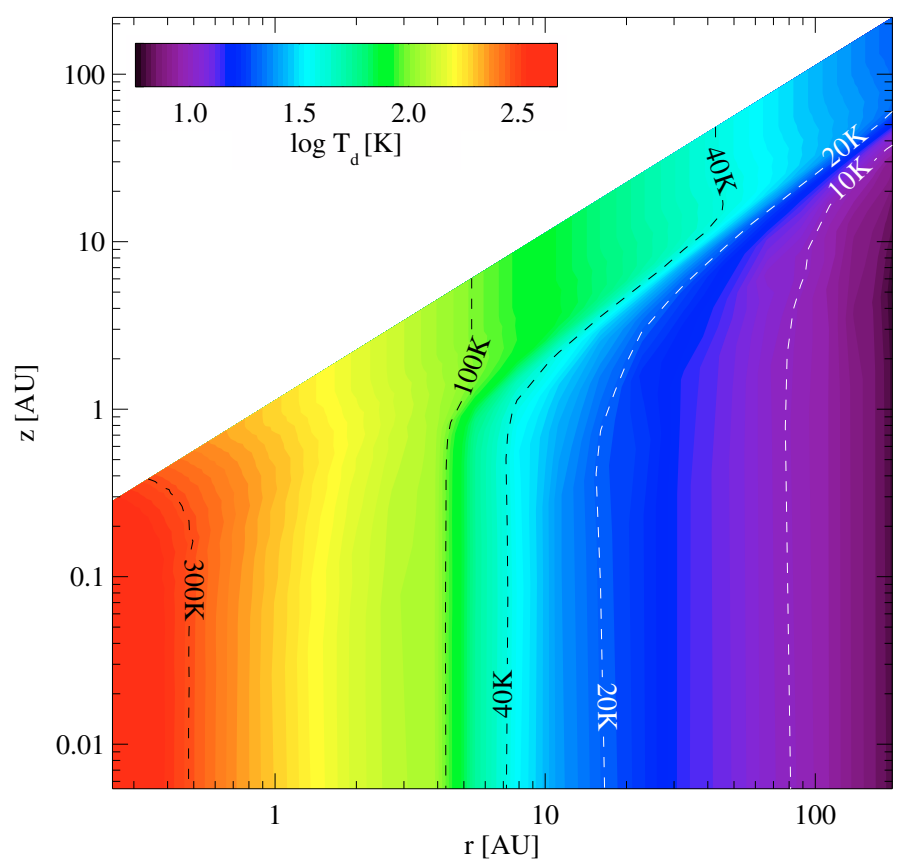

Fig. A.2. Dust temperature profile.

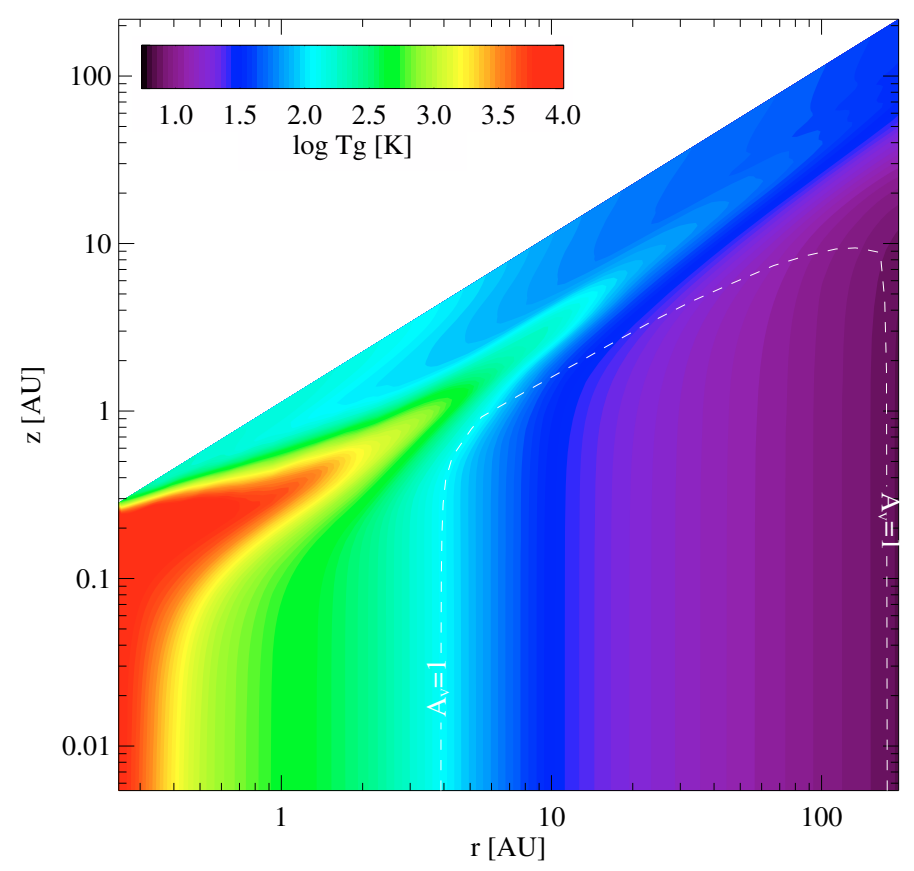

Fig. A.3. Gas temperature profile. The contour of $A_{V}=1$ is shown in white. 